\title{
Recall strategies for patients found to have a nodule in cirrhosis: is there still a role for CEUS?
}

\author{
Vito Cantisani' ${ }^{1}$, Emanuele David ${ }^{1,2}$, Franca Maria Meloni ${ }^{3}$, Christoph Frank Dietrich ${ }^{4}$, \\ Radu Badea ${ }^{5}$, Daniela Messineo ${ }^{1}$, Ferdinando D’Ambrosio1, Fabio Piscaglia ${ }^{6}$
}

${ }^{1}$ Department of Radiology, Oncology, and Anatomy Pathology, “Sapienza” University of Rome, Italy, ${ }^{2}$ Department of Medical Sciences and Morphological and Functional Imaging, Univ. Messina, Messina, Italy, ${ }^{3}$ Dipartimenti servizi diagnostici Ospedale Valduce, Como, Italy ${ }^{4}$ Caritas Krankenhaus Bad Mergentheim, Germany, ${ }^{5}$ Ultrasound Imaging Laboratory, Department of Imaging and Radiology, "Iuliu Hatieganu” University of Medicine and Pharmacy, ClujNapoca, Romania, ${ }^{6}$ Division of Internal Medicine, Department of Medical and Surgical Sciences DIMEC, University of Bologna, S. Orsola-Malpighi Hospital, Bologna, Italy

\begin{abstract}
Development of liver tumors and their evolution to hepatocellular carcinoma (HCC) is a multi-step process in which different HCC-etiologies induce continuous rounds of hepatocyte damage and regeneration. Over an extended time, this triggers cirrhosis which is a pathological state of the liver in which lesions can progress to become dysplastic nodules. Later, these nodules may evolve into HCC and occasionally generate metastatic events. To provide optimal care, patients with liver cancer should be managed using a multidisciplinary approach in specialized centers in which all the diagnostic and therapeutic resources are available. Among the different imaging modalities the introduction on contrast agents for ultrasound use has opened new further applications in different clinical settings. In fact, contrast enhanced ultrasound (CEUS) has been applied for more than ten years and plays increasingly important roles in the management of HCC. Since early 2000, international societies including the American Association for the Study of Liver Diseases (AASLD), the European Association for the Study of the Liver (EASL), the Asian Pacific association for the Study of the Liver (APASL), the Japanese society of Hepatology (JSH), the Italian society for the study of the liver (AISF), the World Federation for Ultrasound in Medicine and Biology (WFUMB), and the European Federation of Societies for Ultrasound in Medicine and Biology (EFUSMB) have discussed the important role of CEUS in the diagnosis of HCC. In the present review an update of the literature and a detailed discussion of the present Guidelines regarding the role of CEUS in the evaluation of nodules in cirrhotic patients is offered.

Keywords: hepatocellular carcinoma, contrast-enhanced ultrasound, CT, MRI, cirrhosis guidelines
\end{abstract}

Liver cirrhosis is defined as the histological development of regenerative nodules surrounded by fibrous bands in response to chronic liver injury such as alcohol, infections, autoimmune, vascular and metabolic dis-

Received 16.09.2015 Accepted 6.10.2015

Med Ultrason

2015, Vol. 17, No 4, 515-520

Corresponding author: Dr. Vito Cantisani, MD, PhD

Dirigente Medico I livello,

Policlinico Umberto i, Univ. Sapienza, Roma

Department of Radiology, Oncology, and

Anatomy Pathology "Sapienza" University of Rome Policlinico Umberto I

Viale del Policlinico 155, 00161 Rome, Italy

E-mail: vito.cantisani@uniroma1.it

Fax: +39 06490243, Phone: +39064455602 eases, biliary obstruction, or cryptogenic forms. It is a pathological state of the liver in which regenerative nodules can progress to become dysplastic nodules. Later, these nodules may evolve into hepatocellular carcinoma (HCC) and occasionally generate metastases [1].

$\mathrm{HCC}$ is the sixth most common malignant tumor in the world and the third cause of cancer-related death. In Western countries it arises in a cirrhotic liver in the large majority of cases $(90 \%)$ although it may occur in some patients with chronic hepatitis B (HBV) or chronic hepatitis $\mathrm{C}(\mathrm{HCV})$ infection, non alcoholic fatty liver disease, or alcohol abuse. The risk of HCC is higher when the nodule size increases. Nodules $<1 \mathrm{~cm}$ are rarely malignant. In nodules $>1 \mathrm{~cm}$ the rate of $\mathrm{HCC}$ is $66 \%$, in 
nodules $1-2 \mathrm{~cm}$ in diameter [2,3], about $80 \%$ in nodules $2-3 \mathrm{~cm}$, and $92 \%-95 \%$ in nodules $>3 \mathrm{~cm} \mathrm{[4].} \mathrm{To} \mathrm{pro-}$ vide optimal care, patients with liver cancer should be managed using a multidisciplinary approach in specialized centers in which all the diagnostic and therapeutic resources are available.

There is evidence that surveillance for HCC can decrease the disease-related mortality [5]. In the western world, surveillance is recommended for subjects at a high risk of developing $\mathrm{HCC}$ such as patients with cirrhosis and certain categories of patients with chronic hepatitis [6], while Japanese guidelines extended this recommendation to all patients with chronic hepatitis [7].

Currently, ultrasonography (US) is the most widely used method for HCC surveillance [8]. According to a recent meta-analysis with 13 studies included by Singal et al [9], the pooled sensitivities and specificities for detecting $\mathrm{HCC}$ at any stage were both $94 \%$. The potential benefit of combining US with alfafetoprotein (AFP) for the detection of early stages HCC was also explored. The pooled sensitivities increased from $63 \%$ to $69 \%$, but it was not statistically significant. For screening HCC in high-risk patients with viral-related cirrhosis or chronic alcoholic liver disease, US at 6-month intervals is recommended by the 2010 American Association for the Study of Liver Diseases (AASLD) guidelines [10]. The European Association for the Study of the Liver (EASL) and the Asian Pacific association for the Study of the Liver (APASL) guidelines recommend surveillance with US, using an interval of 6 months. Moreover, APASL guidelines suggest combining US and AFP measurements $[11,12]$.

Once a hepatic lesion is detected, it is crucial to characterize it in order to confirm or rule out HCC or other malignancies. Although conventional grey scale US is sensitive enough for surveillance of the patients at risk of HCC, as reported above, it presents low accuracy for optimal characterization of focal liver lesions [13].

Although to date, several imaging modalities such as Computed Tomography (CT), Magnetic Resonance Imaging (MRI), PET-CT or positrone emission tomography (PET)-MRI [13] and contrast enhanced ultrasound (CEUS) are available to evaluate these patients within the recall strategy, only CT and MRI are endorsed by international Western guidelines [14].

The introduction of contrast agents for ultrasound use has opened further applications in different clinical settings such as evaluation of lesions in cirrhotic patients. So far, several papers have been published about their role for optimal characterization of hepatic liver lesions as well as for cirrhotic patients. However, to decide about the prompt work-up, usually two possibilities may help us to standardize worldwide clinical practice: one is by evaluating data with multicenter studies on a large number of patients and the second is by applying international guidelines based on systematic literature review. Many international societies have discussed the important role of CEUS in the diagnosis of HCC, since mid 2000, such as the AASLD, EASL, APASL, the Japanese Society of Hepatology (JSH), the Italian Society for the Study of the Liver (AISF), the World Federation for Ultrasound in Medicine and Biology (WFUMB) and the European Federation of Societies for Ultrasound in Medicine and Biology (EFSUMB) $[6,10-12,15,16]$.

When dealing with CEUS with purely blood pool contrast agents, such as sulfur hexafluoride, we have to take in account its peculiar feature: once injected, contrast agents remain confined within the blood territory, thus lacking the extravascular phase. CEUS can therefore depict macro- and microvascularization [17-19]. As a general statement, the occurence of hypoenhancement of solid liver lesions in the late and post-vascular phases signals malignancy with high accuracy in non cirrhotic livers. Conversely, sustained enhancement in the portal and late phases is typically observed in almost all solid benign liver lesions in this setting. However, when dealing with lesions in cirrhosis such patterns are no more highly relevant, but rather the arterial phase becomes of greater significance. The primary feature to be searched in order to to characterize lesions in cirrhosis is the hyperenhancement in the arterial phase although the detection of subsequent hypoenhancement during portal or late phase is requested to definitively establish the diagnosis of HCC [15].

Hyperenhancement in the arterial phase is usually homogeneous and intense in HCC but it may be inhomogeneous, especially in larger nodules $(>5 \mathrm{~cm})$, because of areas of necrosis. The rates of arterial hyperenhancement are variable, increasing with size: in lesions $\leq 2.0$ $\mathrm{cm}$, and $3.0 \mathrm{~cm}$ are between $40-70 \%$ respectively $[2,3]$. Hypoenhancement in the late phase is observed overall in about half of the cases of HCC but more rarely in small nodules $(20 \%-30 \%$ in those $1-2 \mathrm{~cm})$ [3]. It tends to start later in $\mathrm{HCC}$, usually not before $60 \mathrm{~s}$ after injection of the contrast agent and in about $25 \%$ of cases appears only after $180 \mathrm{~s}$; therefore, it is important to prolong the observation of nodules in cirrhosis up to 4 minutes. By applying these features, several authors have shown that CEUS is an effective imaging modality to characterize liver lesions in cirrhosis. According to those results, AASLD included CEUS in the practical guidelines of HCC management in 2005 [10]. In details, they provided the following recommendations: nodules less than $1 \mathrm{~cm}$ should be followed with US every 3-6 months until 2 years and when 
no growth had been observed, routine surveillance may be advised; nodules between 1-2 cm found on US should be investigated further with two dynamic studies, either CT scan, CEUS, or MRI with contrast. If they show typical characteristics of $\mathrm{HCC}$ in two techniques the lesions should be treated as HCC, while in the doubtful case, the lesion should be biopsied If the nodule is larger than 2 $\mathrm{cm}$ and has the typical features of HCC on a dynamic imaging technique or AFP is $>200 \mathrm{ng} / \mathrm{mL}$, a biopsy is not necessary. However, in a doubtful case or if the nodule is detected in a non cirrhotic liver, a biopsy is needed. If the biopsy is negative for HCC, patients should be followed by US or CT scanning at 3-6 monthly intervals until the nodule disappears, enlarges, or displays diagnostic characteristics of $\mathrm{HCC}$.

However, a few years later, Vilana et al reported that intrahepatic cholangiocellular carcinoma (ICC) in cirrhotic liver may not be clearly distinguishable from HCC at CEUS [20]. A total of 21 patients were evaluated. The median nodule size was $32 \mathrm{~mm}$. All nodules showed contrast enhancement in the arterial phase; in 10 cases it was homogeneous and in 11 cases peripheral (rim-like). All nodules displayed washout during the venous phases. In 5 of the 11 nodules showing homogeenous arterial hyperenhancement, wash out had already taken place already during the portal phase and it appeared no earlier than in the late phase in the remaining 6 cases. These 10 nodules (five larger than $2 \mathrm{~cm}$ ) displayed the pattern considered diagnostic for HCC, namely homogeneous contrast uptake followed by washout according to the AASLD criteria. However, none of these lesions displayed wash-out in the venous phase on MRI, leading therefore to a non diagnostic pattern at MRI and hence to biopsy. They concluded that CEUS should not be used as the sole imaging technique for conclusive HCC diagnosis and, if MRI does not confirm the diagnostic vascular pattern in the venous phase, a biopsy is recommended. Following this report, CEUS was dropped from the diagnostic techniques in the latest AASLD Guidelines, as it may offer a false positive HCC diagnosis in patients with ICC [10], and also because CEUS for non-cardiac indications is not available in the USA, so CEUS liver scanning is not applicable for the North American population [21].

However, Barreiros et al also commented that CEUS may be important to identify arterial hypervascularity in presence of a typical pattern of HCC. Accordingly, the diagnosis of HCC should be questioned only when either the wash-out is atypical for HCC (very rapid and/or very marked) or the pattern at CT/MRI for staging is atypical. In fact, rejecting CEUS as a diagnostic technique would lead to more biopsies including the respective risk and less benefit. In addition, ICCs are relatively rare in cir- rhotic livers (1-2\% of all new nodules) and CEUS can even show differences in the vascularization patterns between HCC and ICC [22]. HCC shows an arterial global hyperenhancement and delayed contrast wash-out in the late phase. ICCs are characterized by an arterial contrast enhancement at the tumor periphery with early contrast wash-out of the vascularized parts of the lesions in the portal and late phases [23]. In addition, since the evaluation of cirrhotic patients is performed by applying multimodality imaging strategy, CT and MRI have to be performed. As reported by D'Onofrio et al, the behavior of ICC at CEUS is different from the one in CT. During the late phase, ICC appears hypervascular at CT, because of desmoplastic reaction due to fibrotic component, while at CEUS it appears hypovascular [22]. In these cases CEUS should not be used as the sole imaging technique for conclusive hepatocellular carcinoma diagnosis and if the MRI does not display the diagnostic vascular pattern, a confirmatory biopsy is mandatory [20]. More recently, de Sio et al enrolled 282 cirrhotic patients who underwent CEUS and subsequent biopsy with histological evaluation as the gold standard for correct classification of FLLs. They calculated the best 'time to washout' cut-off values to characterize FLLs. Histological diagnosis of liver lesions was as follows: 34 benign and 248 malignant lesions (223 well-to-moderately differentiated HCCs; 7 poorly-differentiated HCCs; 5 ICCs; 5 primary non-Hodgkin B-cell lymphomas (NHBLs); and 8 metastatic liver tumors). A wash-out time $>55$ s identified patients with HCC with the highest level of accuracy $(92.7 \%)$. Similarly, a wash-out time $\leq 55$ s correctly identified the vast majority of the non-HCC malignancies (100\% sensitivity, $98.2 \%$ specificity and diagnostic accuracy of $98.3 \%$ ). They concluded that CEUS is an accurate and safe procedure for discriminating FLLs in cirrhotic patients, especially when a cut-off wash-out time of $55 \mathrm{~s}$ is chosen as a reference value. Very similar timing for the occurrence of wash-out in ICC (median time around 50 secs) were reported also by another recent Chinese study, which addressed the issue of ICC in cirrhosis [24].

Sporea et al reported their experience based on a total number of 356 cases in which CEUS had $85.7 \%$ sensitivity, $85.9 \%$ specificity, $91.6 \%$ positive predictive value, $77.1 \%$ negative predictive value, and $85.8 \%$ accuracy for differentiation between malignant and benign liver lesions. In particular, they showed that the majority of HCC presented wash-in and later wash-out, while ICC peripheral wash-in and earlier washout. The CEUS accuracy for differentiation between malignant and benign liver lesions was similar in tumors $\leq 2 \mathrm{~cm}$ and those $>2 \mathrm{~cm}$. In this study the reference methods were CT, MRI, or biopsy. These results were in concordance with 
the DEGUM and STIC studies [25]. However, more recently other international guidelines have been published such as WFUMB-EFSUMB and AISF, especially the former includes input from the other professional bodies [15]. These guidelines underline the fact that CEUS is increasingly accepted for clinical diagnostic imaging in focal liver lesions in cirrhosis (FLLs) playing an important role for characterization and post-ablation evaluation. In fact, several studies demonstrated a high sensitivity and specificity in the evaluation of ablation treatments of liver tumors [26,27]. The guidelines point out that CEUS cannot be used to detect - as opposed to characterize - small HCC, since the CEUS identification of small HCC relies on the detection of the hypervascularisation in the arterial vascular phase which is too fast and too transient for the evaluation of the whole liver in the very short arterial enhancement phase. It is recommended that the detection of small HCC must be first carried out using baseline US. These respected guidelines are held in high esteem by many experts who describe in detail the presentation of HCC at CEUS in non-cirrhotic and cirrhotic liver and in variable clinical settings. They report "that CEUS is extremely useful, especially when performed immediately after nodule detection, to make a rapid diagnosis. However, CT or MRI are needed (unless contraindicated) to stage the disease before the treatment strategy is decided". In 2012 an expert panel nominated by the Italian Association for the Study of the Liver (AISF) issued guidelines for a multidisciplinary clinical approach to HCC, with the scope of developing practical recommendations on the multidisciplinary management for the diagnosis of HCC, providing the most appropriate way to define the staging and treatment according to the patient's status and tumor stage at diagnosis. They provided recommendations for an effective diagnostic and staging work-up, followed by recommendations for the best patient-tailored treatment choice. The AISF Expert Panel is based on the 2010 updated AASLD guidelines for HCC. The AISF guidelines state that patients at risk of HCC development should be enrolled in surveillance programs that in most cases consist of bi-annual liver ultrasound. In other cases, i.e. in the presence of conditions clearly limiting the accuracy of ultrasound, CT or MRI may be proposed as a supplementary imaging technique.

CEUS is discussed for the recall and characterization of a focal liver lesion detected at US surveillance. The recall strategy for lesions $\geq 1 \mathrm{~cm}$ is based on contrast-enhanced imaging techniques with the use of vascular contrast media. However, more emphasis was put on either $\mathrm{CT}$ or MRI. The recommendations state that "the lesion should be assessed prior to and after contrast injection in the arterial, portal and venous phases (dynamic contrast imaging) at either CT or MRI'. Diagnosis depends on the vascular pattern of the lesion during contrast media injection in the arterial, portal and venous phases and CEUS is a reliable technique to detect tumor vascularity. The recommendations continue with considerations on the use of CEUS. In the updated AASLD algorithm, CEUS is not included among the imaging techniques for the diagnosis of HCC in a lesion detected during surveillance as it was in the past [16,28]. However, the AISF experts recognize the validity of CEUS since scientific evidence of a number of studies shows that a CEUS wash-in and wash-out pattern typical for HCC has a positive predictive value $>95 \%[16,30]$. AISF experts discuss evidence in the right perspective and they report that ICC accounts for only $1-2 \%$ of all new nodules detected in a cirrhotic liver $[3,29]$ and, among them, only half show the typical HCC pattern at CEUS [28]. Conversely, the AISF expert panel considers the available scientific evidence as insufficient to remove CEUS from the diagnostic tools since a CEUS pattern typical for $\mathrm{HCC}$ has a high positive predictive value. The wash-in/wash-out pattern at CEUS of a nodule in cirrhosis should be considered specific for malignancy and, unless discordant findings with MRI or CT are obtained, it should be considered suggestive of HCC, without the need for biopsy. However, due to the need for CT or MRI for tumor staging, the use of CEUS as a first line approach, despite being possible, is not considered the most cost-effective imaging modality [20]. However, MRI is the gold standard for the characterization of small nodules in cirrhotic liver. The AISF and EFSUMB professional guidelines have not removed CEUS from the management of HCC. While CEUS alone is not recommended for staging, it is still recommended for the evaluation prior to trans-arterial chemoembolization (TACE) or ablation procedures.

Alaboudy et al [30] reported that CEUS combined with CT or MRI improved the sensitivity and specificity of HCC diagnosis.Few articles have focused on the role of preoperative CEUS combined with CE-CT or MRI for staging of HCC in surgical patients, in surgical decisionmaking, or on the correlation between CEUS and intraoperative ultrasound. Even more recently, Zhang et al, evaluated the clinical role of CEUS combined with CE-CT or MRI with the aim to improve the preoperative staging of HCC. Sixty-nine patients who underwent liver resection for HCC were prospectively submitted to CEUS and CECT/MRI before surgery and then to intraoperative ultrasound (IOUS). One hundred and twenty-seven nodules, comprising 94 HCCs confirmed by histopathology and 33 benign lesions confirmed by histopathology and followup, were identified. The overall diagnostic sensitivity rates of CE-CT/MRI, CEUS, IOUS and CEUS + CE-CT/ 
MRI were $78.7 \%, 89.4 \%, 89.4 \%$, and $89.4 \%$, respectively. CEUS combined with CT or MRI significantly increased the diagnostic specificity of CT/MRI, CEUS, and IOUS, alone $(100 \%, 72.7 \%, 97.0 \%$, and $69.7 \%, \mathrm{p}=0.004$, $\mathrm{p}=0.002, \mathrm{p}=0.002$, respectively). The diagnostic accuracy was significantly higher for CEUS + CT/MRI compared with CT/MRI $(92.1 \%$ vs $77.2 \%, \mathrm{p}=0.001)$. Indeed, the authors concluded that CEUS combined with CT or MRI improved the accuracy of preoperative staging for hepatocellular carcinoma and may help to guide individualized treatment for patients with HCC [31].

As showed above, the role of CEUS for the diagnosis of HCC has been a controversial issue, so the technique is continually being evaluated. More recently, a metaanalysis on previous studies to determine the diagnostic accuracy of CEUS for diagnosis of small $(\leq 2 \mathrm{~cm}$ in diameter) HCC was published [32]. A comprehensive literature search of PubMed, Embase, Web of Science, and China BioMedicine databases was conducted on articles published before 1 March 2013; 15 studies were included with a total of 908 cirrhotic patients with 1,032 small hepatic nodules. All lesions were histologically confirmed through liver biopsies after CEUS. The pooled sensitivity was $0.81(95 \% \mathrm{CI}=0.78-0.85)$, the pooled specificity was $0.86(95 \% \mathrm{CI}=0.82-0.89)$, the pooled positive LR was $5.90(95 \% \mathrm{CI}=3.90-8.94)$, the pooled negative LR was $0.20(95 \% \mathrm{CI}=0.14-0.29)$, and the pooled DOR was 37.07 (95\% CI=24.79-55.44). According to this metaanalysis, CEUS is a useful diagnostic tool with high sensitivity and specificity for identifying small HCC [32].

Therefore, it is generally accepted that CEUS is a cost-effective second line imaging modality once the liver focal lesion is detected at US although MRI is the gold standard for the characterization of small nodules at high-risk for HCC in cirrhotic liver. However, CEUS as recently reported by Jang et al [33], has some advantages over CT and MRI such as: the more sensitive depiction of arterial hypervascularity of HCC, the better demonstration of rapid wash-out for non-HCC malignancy, as well as of very late wash-out of HCC. Visualization of early vascular filling patterns for benign hypervascular lesions is of indisputable value. A frequently unaccounted benefit of CEUS includes the value of its performance following nodule detection at ultrasound surveillance, including one-stop exclusion of typical benignancy, preclusion of arterial pseudolesions shown on CT/MR, and the avoidance of miscorrelation of a nodule on surveillance and subsequent diagnostic imaging. Therefore, CEUS can effectively be used in the diagnostic algorithm for new liver nodules detected during HCC surveillance.

In conclusion there has been a lot of controversy regarding the role of CEUS for the diagnosis of HCC, and the technique is continually being evaluated. In our opinion, the role of CEUS in HCC management will be re-considered as technology (e.g. quantitative perfusion) continues to evolve and as its use spreads not only among expert operators and referral centers but also among everyday ultrasound personnel involved in HCC surveillance [34].

Conflict of interest: We want to mention that Vito Cantisani, lectured for Bracco SpA Milan, Samsung, Toshiba, and Siemens, Franca Meloni for Bracco, Fabio Piscaglia was a speaker and advisory board member for Bayer, a speaker for Bracco and Siemens and he is on the Advisory board for GE and has a research contract with Esaote. All the aforementioned activities do not represent potential conflict of interest

\section{References}

1. Bisteau X, Caldez MJ, Kaldis P. The Complex Relationship between Liver Cancer and the Cell Cycle: A Story of Multiple Regulations. Cancers (Basel) 2014; 6: 79-111.

2. Forner A, Vilana R, Ayuso C, et al. Diagnosis of hepatic nodules $20 \mathrm{~mm}$ or smaller in cirrhosis: Prospective validation of the noninvasive diagnostic criteria for hepatocellular carcinoma. Hepatology 2008; 47: 97-104.

3. Sangiovanni A, Manini MA, Iavarone M, et al. The diagnostic and economic impact of contrast imaging techniques in the diagnosis of small hepatocellular carcinoma in cirrhosis. Gut 2010; 59: 638-644.

4. Bolondi L, Sofia S, Siringo S, et al. Surveillance programme of cirrhotic patients for early diagnosis and treatment of hepatocellular carcinoma: a cost effectiveness analysis. Gut 2001; 48: 251-259.

5. Cucchetti A, Trevisani F, Pecorelli A, et al. Estimation of lead-time bias and its impact on the outcome of surveillance for the early diagnosis of hepatocellular carcinoma. J Hepatol 2014; 61: 333-341.

6. Italian Association for the Study of the Liver (AISF); AISF Expert Panel; AISF Coordinating Committee; et al. Position paper of the Italian Association for the Study of the Liver (AISF): the multidisciplinary clinical approach to hepatocellular carcinoma. Dig Liver Dis 2013; 45: 712-723.

7. Giannini EG, Cucchetti A, Erroi V, Garuti F, Odaldi F, Trevisani F. Surveillance for early diagnosis of hepatocellular carcinoma: how best to do it? World J Gastroenterol 2013; 19: 8808-8821.

8. van Meer S, de Man RA, Siersema PD, van Erpecum KJ. Surveillance for hepatocellular carcinoma in chronic liver disease: evidence and controversies. World J Gastroenterol 2013; 19: 6744-6756.

9. Singal A, Volk ML, Waljee A, Salgia R, Higgins P, Rogers MA, Marrero JA. Meta-analysis: surveillance with ultrasound for early-stage hepatocellular carcinoma in patients with cirrhosis. Aliment Pharmacol Ther 2009; 30: 37-47. 
10. Bruix J, Sherman M; American Association for the Study of Liver D. Management of hepatocellular carcinoma: an update. Hepatology 2011; 53: 1020-1022.

11. European Association For The Study Of The Liver, European Organisation For Research And Treatment Of Cancer. EASL-EORTC clinical practice guidelines: management of hepatocellular carcinoma. J Hepatol 2012; 56: 908-943.

12. Omata M, Lesmana LA, Tateishi R, et al. Asian Pacific Association for the Study of the Liver consensus recommendations on hepatocellular carcinoma. Hepatol Int 2010; 4: 439-474.

13. de Sio I, Iadevaia MD, Vitale LM, et al. Optimized contrast-enhanced ultrasonography for characterization of focal liver lesions in cirrhosis: A single-center retrospective study. United European Gastroenterol J 2014; 2: 279-287.

14. Bruix J, Reig M, Rimola J, et al. Clinical decision making and research in hepatocellular carcinoma: pivotal role of imaging techniques. Hepatology 2011; 54: 2238-2244.

15. Claudon M, Dietrich CF, Choi BI, et al. Guidelines and good clinical practice recommendations for Contrast Enhanced Ultrasound (CEUS) in the liver - update 2012: A WFUMB-EFSUMB initiative in cooperation with representatives of AFSUMB, AIUM, ASUM, FLAUS and ICUS. Ultrasound Med Biol 2013; 39: 187-210.

16. Kudo M, Izumi N, Kokudo N, et al. Management of hepatocellular carcinoma in Japan: Consensus-Based Clinical Practice Guidelines proposed by the Japan Society of Hepatology (JSH) 2010 updated version. Dig Dis 2011; 29: 339-364.

17. Chiorean L, Cantisani V, Jenssen C, Sidhu PS, Baum U, Dietrich CF. Focal masses in a non-cirrhotic liver: The additional benefit of CEUS over baseline imaging. Eur J Radiol 2015; 84: 1636-1643.

18. Ricci P, Laghi A, Cantisani V, et al. Contrast-enhanced sonography with SonoVue: enhancement patterns of benign focal liver lesions and correlation with dynamic gadobenate dimeglumine-enhanced MRI. AJR Am J Roentgenol 2005; 184: 821-827.

19. Friedrich-Rust M, Klopffleisch T, Nierhoff J, et al. Contrast-Enhanced Ultrasound for the differentiation of benign and malignant focal liver lesions: a meta-analysis. Liver Int 2013; 33: 739-755.

20. Vilana R, Forner A, Bianchi L, et al. Intrahepatic peripheral cholangiocarcinoma in cirrhosis patients may display a vascular pattern similar to hepatocellular carcinoma on contrastenhanced ultrasound. Hepatology 2010; 51: 2020-2029.

21. Galassi M, Iavarone M, Rossi S, et al. Patterns of appearance and risk of misdiagnosis of intrahepatic cholangiocarcinoma in cirrhosis at contrast enhanced ultrasound. Liver Int 2013; 33: 771-779.

22. Wildner D, Bernatik T, Greis C, Seitz K, Neurath MF, Strobel D. CEUS in hepatocellular carcinoma and intrahepatic cholangiocellular carcinoma in 320 patients - early or late washout matters: a subanalysis of the DEGUM multicenter trial. Ultraschall Med 2015; 36: 132-139.

23. D'Onofrio M, Vecchiato F, Cantisani V, et al. Intrahepatic peripheral cholangiocarcinoma (IPCC): comparison between perfusion ultrasound and CT imaging. Radiol Med 2008; 113: 76-86.

24. Yuan MX, Li R, Zhang XH, et al. Factors Affecting the Enhancement Patterns of Intrahepatic Cholangiocarcinoma (ICC) on Contrast-Enhanced Ultrasound (CEUS) and their Pathological Correlations in Patients with a Single Lesion. Ultraschall Med 2015. doi: 10.1055/s-0034-1399485.

25. Sporea I, Badea R, Popescu A, et al. Contrast-enhanced ultrasound (CEUS) for the evaluation of focal liver lesions - a prospective multicenter study of its usefulness in clinical practice. Ultraschall Med 2014; 35: 259-266.

26. Ricci P, Cantisani V, Drudi F, et al. Is contrast-enhanced US alternative to spiral CT in the assessment of treatment outcome of radiofrequency ablation in hepatocellular carcinoma? Ultraschall Med 2009; 30: 252-258.

27. Meloni MF, Smolock A, Cantisani V, et al. Contrast enhanced ultrasound in the evaluation and percutaneous treatment of hepatic and renal tumors. Eur J Radiol 2015; 84: 1666-1674.

28. Chen LD, Xu HX, Xie XY, et al. Intrahepatic cholangiocarcinoma and hepatocellular carcinoma: differential diagnosis with contrast-enhanced ultrasound. Eur Radiol 2010; 20: 743-753.

29. Boozari B, Soudah B, Rifai K, et al. Grading of hypervascular hepatocellular carcinoma using late phase of contrast enhanced sonography - a prospective study. Dig Liver Dis 2011; 43: 484-490.

30. Alaboudy A, Inoue T, Hatanaka K, et al. Usefulness of combination of imaging modalities in the diagnosis of hepatocellular carcinoma using Sonazoid $\AA$-enhanced ultrasound, gadolinium diethylene-triamine-pentaacetic acid-enhanced magnetic resonance imaging, and contrast-enhanced computed tomography. Oncology 2011; 81 Suppl 1: 66-72.

31. Zhang XY, Luo Y, Wen TF, et al. Contrast-enhanced ultrasound: Improving the preoperative staging of hepatocellular carcinoma and guiding individual treatment. World $\mathrm{J}$ Gastroenterol 2014; 20: 12628-12636.

32. Niu Y, Huang T, Lian F, Li F. Contrast-enhanced ultrasonography for the diagnosis of small hepatocellular carcinoma: a meta-analysis and meta-regression analysis. Tumour Biol 2013; 34: 3667-3674.

33. Jang HJ, Kim TK, Burns PN, Wilson SR. CEUS: An essential component in a multimodality approach to small nodules in patients at high-risk for hepatocellular carcinoma. Eur J Radiol. 2015; 84: 1623-1635.

34. Wilson SR, Kim TK, Jang HJ, Burns PN. Enhancement patterns of focal liver masses: discordance between contrastenhanced sonography and contrast-enhanced CT and MRI. AJR Am J Roentgenol 2007; 189: W7-W12. 